\title{
Symmetry Rules: How Science and Nature Are Founded on Symmetry. By Joe Rosen. Springer: Berlin. 2008, XIV, 305 p. 86 illus., Hardcover. CHF 70. ISBN: 978-3-540-75972-0
}

\section{Shu-Kun Lin}

Molecular Diversity Preservation International (MDPI), Matthaeusstrasse 11, CH-4057 Basel, Switzerland; Tel. (+41) 79322 3379; Fax: (+41) 61302 8918; E-mail: lin@mdpi.org; http://www.mdpi.org/lin

Received: 14 April 2008 / Published: 16 June 2008

This book belongs to the book series The Frontiers Collection, edited by A.C. Elitzur, M.P. Silverman, J. Tuszynski, R. Vaas and H.D. Zeh.

In Prigogine's entropy theory symmetry has been regarded as "order" (reduction of entropy). The main idea of the Chemistry Nobel Prize winner Prigogine's thermodynamic theory cannot be correct, because as a senior chemist I know Prigogine's theory has not found any application in chemistry so far. Joe Rosen's symmetry principle is the opposite of Prigogine's theory. Recently more and more chemists and other scientists have started to apply Rosen's symmetry principle and cite his symmetry principle books. The symmetry principle can be readily applied to all chemical processes, without exception. The Curie symmetry principle has been greatly developed by Rosen, whose symmetry principle states that the symmetry group of the cause is a subgroup of the symmetry group of the effect [13]. Symmetry rules related to Rosen's symmetry principle are summarized in the last chapter, chapter 12, of this new book.

Rosen also provided proof of the symmetry principle. I have been interested in the symmetryentropy relationship presented in the earlier versions of Joe's books on symmetry principle $[3,4]$. Based on the Gibbs inequality

$$
\ln w \geq-\sum_{i=1}^{w} p_{i} \ln p_{i}
$$

I have proved both the similarity principle (a continuous higher similarity-higher entropy relation after the rejection of the Gibbs paradox) and the Rosen symmetry principle (a higher symmetry-higher stability relation) as a special case of the similarity principle [4].

In chapter 1 and in the other chapters the concepts of symmetry and asymmetry are discussed. Rosen prefers to use "nonsymmetry" instead of "asymmetry" when the Curie's "asymmetry is the cause of the effect" is discussed [5]. For this reason, I changed "asymmetry" to "nonsymmetry" everywhere in the final version of my paper [4]. Obviously I have been influenced by Rosen's books. 
Symmetry and the frame of reference have been discussed more than once in this book. The threedimensional Cartesian coordinate system with $x$-axis, $y$-axis and $z$-axis has asymmetry or chirality. I still remember that the direction of the cross product of two vectors and the direction associated with a rotation were confusing when I was reading literature on NMR (Nuclear magnetic resonance) and EPR (Electron Paramagnetic Resonance), because some authors draw the Cartesian coordinate system in an opposite way. The concept of asymmetry and the definition of symmetry are presented with new insights in this chapter.

Chapters 2 and 3 present the topic of science as founded on symmetry and symmetry in physics. For example, suppose there is another universe. One can predict that $1+1=2$ is still definitely true and many laws of our universe would remain unchanged there, even though I may predict that the speed of light can be a different value if we bring our ruler to that universe and measure (it might be interesting to consider the interaction and communication between the two universes where light travels in different speed). Rosen made interesting remarks on "the arrow of time" [6]:

There is no problem of "the arrow of time". There simply is no arrow of time, as if time could go one "way" rather than another. That metaphor is an unfortunate result of spatializing time. The picture of time as a line along which one might travel in one direction or the other is a conceptual disaster. Time is becoming. Becoming is change. The undoing of a change is also a change. There is no "unbecoming".

This might be true in another universe. It is also nuisance that in another universe the time runs backward. Many people would still remember Prigogine's different ideas about "the arrow of time".

In section 3.2, the topic of symmetry of states as presented in many thick tomes is discussed briefly. As already commented [3], the unique feature of Rosen's books on symmetry is that they mainly discuss the symmetry evolution and processes. Another unique feature is that asymmetry and symmetry aspects is always elegantly considered together.

A completely symmetric universe would be extremely boring. On page 49, an example of such a universe is given as "a universe consisted solely of photons and gravitons, which are respective antiparticles of themselves". When particle and antiparticle encounter, in the word of Curie, some immediate and interesting phenomena will happen, though a mixture of particles and antiparticles is very unstable. A mixture of a cylinder of oxygen and a cylinder of natural gas can supply a lot of (chemical) energy. A mixture of particles and antiparticles will provide cleanest energy with the best efficiency.

Authors interested in "continuous symmetry" concept will find the discussions about "analogy and classification are symmetry" interesting. Joe Rosen actually published papers many years ago on the topic of "continuous symmetry”. Partial symmetry, limited symmetry or approximate symmetry are mentioned on page 43.

Let me stop here. Enjoy reading this book!

Acknowledgments: I am grateful to my long time colleague Dr. Derek McPhee for his collaboration and assistance. 


\section{References and Notes}

1. This book’s homepage: http://www.springer.com/physics/book/978-3-540-75972-0.

2. Rosen, J. Comment: The Symmetry Principle. Entropy 2005, 7, 308-313.

3. Lin, S.-K. Book Review: Symmetry in Science: An Introduction to the General Theory. By Joseph Rosen. Springer Verlag: New York. 1996. 234pp. \$29.95. ISBN 0-387-94836-8. Entropy 1999, 1, 53-54.

4. Lin, S.-K. The Nature of the Chemical Process. 1. Symmetry Evolution - Revised Information Theory, Similarity Principle and Ugly Symmetry. Int. J. Mol. Sci. 2001, 2, 10-39.

5. Rosen, J. Symmetry in Physics: Selected Reprints, American Association of Physics Teachers: Stony Brook, New York, 1982.

6. Rosen, J. Time, c, and nonlocality: A glimpse beneath the surface? Physics Essays 1994, 7, 335340.

(C) 2008 by the authors; licensee Molecular Diversity Preservation International, Basel, Switzerland. This article is an open-access article distributed under the terms and conditions of the Creative Commons Attribution license (http://creativecommons.org/licenses/by/3.0/). 